\title{
Diabetes digital app technology: benefits, challenges, and recommendations. A consensus report by the European Association for the Study of Diabetes (EASD) and the American Diabetes Association (ADA) Diabetes Technology Working Group
}

\author{
G. Alexander Fleming ${ }^{1} \cdot$ John R. Petrie ${ }^{2} \cdot$ Richard M. Bergenstal $^{3} \cdot$ Reinhard W. Holl $^{4} \cdot$ Anne L. Peters $^{5}$. \\ Lutz Heinemann ${ }^{6}$
}

Published online: 5 December 2019

(C) European Association for the Study of Diabetes and American Diabetes Association 2019

\begin{abstract}
Digital health technology, especially digital and health applications ('apps'), have been developing rapidly to help people manage their diabetes. Numerous health-related apps provided on smartphones and other wireless devices are available to support people with diabetes who need to adopt either lifestyle interventions or medication adjustments in response to glucose-monitoring data. However, regulations and guidelines have not caught up with the burgeoning field to standardise how mobile health apps are reviewed and monitored for patient safety and clinical validity. The available evidence on the safety and effectiveness of mobile health apps, especially for diabetes, remains limited. The European Association for the Study of Diabetes (EASD) and the American Diabetes Association (ADA) have therefore conducted a joint review of the current landscape of available diabetes digital health technology (only stand-alone diabetes apps, as opposed to those that are integral to a regulated medical device, such as insulin pumps, continuous glucose monitoring systems, and automated insulin delivery systems) and practices of regulatory authorities and organisations. We found that, across the USA and Europe, mobile apps intended to manage health and wellness are largely unregulated unless they meet the definition of medical devices for therapeutic and/or diagnostic purposes. International organisations, including the International Medical Device Regulators Forum and WHO, have made strides in classifying different types of digital health technology and integrating digital health technology into the field of medical devices. As the diabetes digital health field continues to develop and become more fully integrated into everyday life, we wish to ensure that it is based on the best evidence for safety and efficacy. As a result, we bring to light several issues that the diabetes community, including regulatory authorities, policymakers, professional organisations, researchers, people with diabetes and healthcare professionals, needs to address to ensure that diabetes health technology can meet its full potential. These issues range from inadequate evidence on app accuracy and clinical validity to lack of training provision, poor interoperability and standardisation, and insufficient data security. We conclude with a series of recommended actions to resolve some of these shortcomings.
\end{abstract}

Keywords CE mark · Diabetes therapy $\cdot$ Digital apps $\cdot$ Digital health technology $\cdot$ Medical devices $\cdot$ Regulatory agencies · Smartphones

This article is being simultaneously published in Diabetologia (https://doi. org/10.1007/s00125-019-05034-1) and Diabetes Care (https://doi.org/10. 2337/dci19-0062) by the European Association for the Study of Diabetes and the American Diabetes Association.

G. Alexander Fleming

zanfleming@kinexum.com

Kinexum, PO Box 1260, Harpers Ferry, WV 25425, USA

2 Institute of Cardiovascular and Medical Sciences, University of Glasgow, Glasgow, UK
3 International Diabetes Center at Park Nicollet, Minneapolis, MN, USA

4 Institute of Epidemiology and Medical Biometry, ZIBMT, University of Ulm, Ulm, Germany

5 Keck School of Medicine of the University of Southern California, Los Angeles, CA, USA

6 Science-Consulting in Diabetes GmbH, Neuss, Germany 


$\begin{array}{ll}\text { Abbreviations } \\ \text { AID } & \text { Automated insulin delivery } \\ \text { BGM } & \text { Blood glucose monitoring } \\ \text { CE } & \text { Conformité Européenne } \\ \text { CGM } & \text { Continuous glucose monitoring } \\ \text { DTSec } & \text { Standard for Wireless Diabetes Device Security } \\ \text { EMA } & \text { European Medicines Agency } \\ \text { EU } & \text { European Union } \\ \text { FDA } & \text { Food and Drug Administration } \\ \text { FDCA } & \text { Federal Food, Drug and Cosmetic Act } \\ \text { GOe } & \text { Global Observatory for eHealth } \\ \text { HCP } & \text { Healthcare professional } \\ \text { IMDRF } & \text { International Medical Device Regulators Forum } \\ \text { MDR } & \text { Medical Device Regulation } \\ \text { mHealth } & \text { Mobile health } \\ \text { NEST } & \text { National Evaluation System for health Technology } \\ \text { NHS } & \text { National Health Service } \\ \text { Pre-Cert } & \text { Precertification Pilot Program } \\ \text { RWPA } & \text { Real-world performance analytics } \\ \text { SaMD } & \text { Software as a Medical Device }\end{array}$

\section{Introduction}

Coincident with the diabetes pandemic of the last three decades has been a revolution in digital and wireless technology [1]. These technological advances have been harnessed to support lifestyle and pharmacological interventions, as well as medical devices (blood glucose meters, continuous glucose monitoring [CGM] devices, insulin pumps and smart pens) [2-5]. At the forefront is the burgeoning field of digital health technology, notably digital health apps, for people with or at risk for diabetes, which has proliferated and begun to permeate clinical care, research and health product development [6].

This position statement focuses on digital health apps. Digital health, also known as mobile health ('mHealth'), is defined by the WHO Global Observatory for eHealth (GOe) as 'medical and public health practice supported by mobile devices, such as mobile phones, patient monitoring devices, personal digital assistants (PDAs), and other wireless devices' [7]. Digital health apps can be generally broken down into three categories: those used for tracking wellness, those that function as stand-alone medical devices (e.g. for titrating insulin), and those that display, download and/or use data from medical devices that diagnose, prevent, monitor or treat a condition (e.g. blood glucose monitoring [BGM], CGM, insulin pump or automated insulin delivery [AID] system [also known as 'closed loop' control system]) [8]. Among almost half a million health-related apps available for wireless devices (usually smartphones) [9-10], apps designed to help manage diabetes are among those most commonly available [11]. These are intended to improve health outcomes and quality of life by coaching people with diabetes, supporting healthy nutrition and weight control, encouraging glucose monitoring and remote monitoring, assisting with the interpretation of results, maintaining lifestyle modifications, guiding medication dosing and, ultimately, reducing complications [12]. Due to the vastness of the field of digital health apps, this position statement will go into discussion of only stand-alone apps that are not integral to a regulated medical device. Examples of what is out of scope of this position statement include insulin pumps and AID systems.

Table 1 lists examples of digital health apps used for managing diabetes according to their intended purpose. It is important to note that many of these apps have more than one feature, and not all are solely for managing diabetes. Earlier in 2019, Kebede and Pischke conducted a study that aimed to identify the most popular diabetes apps via a web-based survey among people with diabetes on social media [13].

Diabetes apps have enormous potential, given that more than 2.7 billion individuals in the world use smartphones [25] and about 0.5 billion people already use mobile apps for diet, physical activity and chronic disease management [26]. Small-scale studies of digital programs targeting glucose control, medication adherence, weight loss and quality of life have shown promising results [27-30]. However, longer-term clinical evidence is needed to more accurately assess the effectiveness of diabetes apps. Currently, many apps are 'stand alone'; however, there is an increasing trend towards integration and increased automation (both in data collection and algorithm-based response). As this trend gains momentum, the landscape of apps is likely to be transformed towards greater integration.

The market-driven explosion of health apps has been facilitated by current systems of regulation. However, not every app is useful or good. Our intention is not to slow growth, but, rather, to make a realistic assessment of what is safe and truly beneficial for people with diabetes. There are very few data on long-term benefits, and even high-quality short-term data are limited [31]. While apps may benefit those with the technical, literacy and numeracy skills to interact with them, many people with diabetes (even in high-income countries) still lack access to healthcare and medications (including insulin) required to sustain life, which may represent more pressing problems to address.

The Diabetes Technology Working Group of the American Diabetes Association (ADA) and the European Association for the Study of Diabetes (EASD) aims to complement already published reviews, position statements, and guidelines on digital health apps [32-35] by reviewing their benefits and risks while providing approaches to handle the challenges they pose. In the remainder of this article, we cover only stand-alone diabetes apps, as opposed to those that are integral to a regulated medical device (e.g. insulin pump, CGM system, AID system). Other topics not covered here that warrant future attention are apps specific to gathering clinical evidence, and apps that support general electronic medical record (EMR) systems. 
Table 1 Types of digital health apps used for managing diabetes

\begin{tabular}{|c|c|c|}
\hline Category name & Description/definition & Examples \\
\hline 1. Nutrition apps & $\begin{array}{l}\text { - Offer databases where users can look up } \\
\text { carbohydrate, fat, protein and energy content } \\
\text { - Support meal planning and insulin dose } \\
\text { adjustment [14] }\end{array}$ & $\begin{array}{l}\text { Carbs and Cals } \\
\text { CarbControl } \\
\text { Foodily } \\
\text { Healthy } \\
\text { Low Carb Program }\end{array}$ \\
\hline 2. Physical activity apps & $\begin{array}{l}\text { - Allow users to track their activity, count } \\
\text { calories and set goals for exercise and } \\
\text { weight management [15] }\end{array}$ & $\begin{array}{l}\text { My Fitness Pal } \\
\text { Nike + Running } \\
\text { Track } 3\end{array}$ \\
\hline 3. Glucose monitoring apps & $\begin{array}{l}\text { - Log glucose data, typically from an external } \\
\text { device that measures glucose (e.g. BGM, CGM) } \\
\text { - Graphically display glucose levels to assist } \\
\text { the patient and HCPs with management of } \\
\text { glucose control }\end{array}$ & $\begin{array}{l}\text { Dexcom Share } \\
\text { Diabetic } \\
\text { Diabetes Companion Diabetes in Check } \\
\text { Glooko Mobile App } \\
\text { Tidepool Mobile }\end{array}$ \\
\hline 4. Insulin titration apps & $\begin{array}{l}\text { - An extension of (3) that also integrate bolus } \\
\text { calculators with traditional blood glucose } \\
\text { meters to help people with diabetes calculate } \\
\text { their basal, prandial and correction insulin } \\
\text { doses [14] }\end{array}$ & $\begin{array}{l}\text { FDA-cleared apps: WellDoc BlueStar [16], } \\
\text { Voluntis Insulia, Sanofi MyDose Coach, } \\
\text { Glooko Mobile Insulin Dosing System, } \\
\text { Amalgam iSage Rx [17] and Hygieia } \\
\text { d-Nav Insulin Guidance System [18] }\end{array}$ \\
\hline 5. Insulin delivery apps & $\begin{array}{l}\text { - For insulin pumps and smart pens to collect and } \\
\text { display data; includes bolus calculators, data } \\
\text { downloaders and firmware update apps [19] } \\
\text { - Such apps also provide decision support }\end{array}$ & $\begin{array}{l}\text { Companion Medical InPen connects to its } \\
\text { smartphone app via Bluetooth } ® \text { to keep } \\
\text { track of insulin data [20] } \\
\text { Dexcom Clarity sends weekly summaries } \\
\text { and pattern identification [21] } \\
\text { Medtronic's Sugar.IQ integrates BGM and } \\
\text { insulin dosing analysis in close to real } \\
\text { time [22] }\end{array}$ \\
\hline $\begin{array}{l}\text { AID systems (also known as closed- } \\
\text { loop control systems, artificial } \\
\text { pancreas systems or autonomous } \\
\text { system for glycaemic control) }\end{array}$ & $\begin{array}{l}\text { - Consists of a CGM system, insulin infusion pump, } \\
\text { and a computer-controlled algorithm (for do-it-yourself } \\
\text { AID systems a smartphone app) to allow } \\
\text { communication between the CGM system and insulin } \\
\text { pump on the patient [23] }\end{array}$ & $\begin{array}{l}\text { Medtronic's MiniMed } 670 \mathrm{G} / \text { Guardian } \\
\text { Sensor } 3 \text { is the first FDA-approved } \\
\text { hybrid AID system that automates basal } \\
\text { insulin infusion rate (still requires meal } \\
\text { boluses) [24] }\end{array}$ \\
\hline
\end{tabular}

\section{The role of regulators}

While most stakeholders in Europe and North America have a general understanding of the approval and regulatory processes governing pharmaceuticals and medical devices, our experience is that levels of awareness of these issues in relation to digital apps are lower. We believe it is important for people with diabetes, as well as healthcare professionals (HCPs), to understand aspects of diabetes app regulation.

European Medicines Agency (EMA) The European Commission has recognised the growing digital health market. In 2012, it released guidance (updated in 2016) on the qualification and classification of stand-alone software used in the healthcare setting as a medical device [36]. Under this guidance, mobile apps are considered medical devices if they are used 'specifically for diagnostic and/or therapeutic purposes', including the diagnosis, prevention, monitoring, treatment or alleviation of disease. In 2014 (and updated in 2018), the Communication on Digital Transformation of Health and Care in the Digital Single Market was published, listing three priorities [37]:
1. Enable citizens to access their health data across the European Union (EU);

2. Allow researchers and other professionals to pool health data across the EU to advance research and personalised health; and

3. Use digital tools to empower people with diabetes to look after their health, prevent diseases and enable feedback and interaction between users and HCPs.

While the European authorities and the US Food and Drug Administration (FDA) share the broad principles of regulating both traditional health products and software, there are substantial differences in the organisational structure of medical product and software registration. The EMA and the FDA are each responsible for pharmaceutical regulation, but only the FDA regulates both pharmaceuticals and medical devices. In the EU, no single agency but the European Commission is responsible for regulation of medical devices; each individual country retains primary responsibility for organising and delivering health services and medical care. As a result, EU member states maintain their own national pharmaceutical regulatory 
authorities, with the European Commission serving to complement national policies and ensure health protection according to EU policies (e.g. the new Medical Device Regulation [MDR]) [38]. Instead, these responsibilities are retained by individual member states, which delegate to accredited notified bodies responsibilities for implementing these regulations. These entities are accredited by the EU to assess whether a product meets the standards set by the EU Medical Devices Directive (MDD), and their decision is valid across all member states. Assessments are based on evidence of safety and performance (in contrast, the FDA may also require clinical effectiveness data, especially for 'high risk devices' (see classification of medical devices in [32]) [39]. If these standards are met, then a manufacturer is authorised to market the product throughout the EU and label it with the Conformité Européenne (CE) mark [40].

In general, the process of obtaining a CE mark in the EU in the past has been a lower hurdle than obtaining device approval or clearance by the FDA [41]. This difference between the USA and EU is likely to narrow with the implementation of the European Union's MDR, which repeals the existing directives on medical devices. The regulation was published on 5 May 2017 and allows a transition time of 3 years before coming into force on 25 May 2020. Currently approved medical devices will have up to 26 May 2020 to meet the new MDR requirements. Among the provisions in this set of regulations are the strengthening of post-market surveillance, establishment of a comprehensive EU database on medical devices, stricter control for high-risk devices before launch in the marketplace, and a new risk classification system for in vitro diagnostic medical devices in line with international guidance [42].

The guidelines in individual countries align with those issued by the European Commission. For example, Sweden's Läkemedelsverket Medical Products Agency classifies medical software as a medical device if its stated purpose complies with the definition in Article 1 of Directive 93/42/EEC on medical devices ('used specifically for diagnostic and/or therapeutic purposes'), has a demonstrated benefit over risk, and is CE-marked [43]. In Germany, medical apps are classified as medical devices if they follow EU guidelines and the German Medical Devices Act and are CEmarked [44]. The situation in the UK was previously similar but is currently in flux because of continuing uncertainties about the UK's relationship with the EU.

More recently, the European Commission has made considerable efforts to introduce and implement the MDR as a new regulatory framework, which will provide clarity on what is (and what is not) a medical device software [42].
FDA With a view to prioritising its resources in the face of an explosive growth of digital health apps, the FDA has attempted to draw a line between those that do and do not require regulation. In 2015, it released a guidance document (updated in 2019) for mobile medical applications for apps that meet the definition of a device in section 201(h) of the Federal Food, Drug and Cosmetic Act (FDCA) [32]. This definition covers apps intended to be used as accessories to regulated medical devices and those that are stand-alone software. However, the guidance expressed its intention to exercise 'enforcement discretion' over those judged to pose a lower risk to users (e.g. apps that provide people with diabetes encouragement to meet their health goals or provide them with tools to track their health information). Thus, using this 'riskbased' approach, mobile apps that calculate insulin doses are within the scope of regulation, while apps that simply organise and/or provide health or nutritional information are not. The FDA lists approved/cleared apps in its $510(\mathrm{k})$ and premarket approval (PMA) databases and on its Registration \& Listing Database [45].

These guidelines were updated when the US Congress passed the 2016 21st Century Cures Act, which specifically excludes from the definition of 'medical device' certain lowrisk medical software. Examples of exclusions from regulation as a medical device include software that supports administrative functions, encourages a healthy lifestyle, serves as an electronic patient record, assists in displaying or storing data, or provides limited clinical data support [46-47]. By the end of 2019, the FDA will launch version 1.0 of the National Evaluation System for health Technology (NEST) initiative, which will be coordinated by the NEST Coordinating Center (NESTcc) [48]. NEST will improve access to evidence across the total product life cycle of medical devices by strategically and systematically leveraging real-world evidence generated by participating institutions and applying advanced analytics tailored to the unique data needs and innovation cycles of medical devices [49]. Using a neural network data model that will represent nearly 500 million patient records from approximately 200 hospitals and 4000 outpatient clinics, this initiative seems promising for medical device stakeholders, especially if it will capture substantial data on people with diabetes.

An important distinction is the difference in the regulation of mobile health apps from the regulation of digital therapeutics (sometimes referred to as 'digiceuticals'). Digital therapeutics are clinically validated digital, usually online, health technologies intended to treat a medical or psychological condition [50]. These are governed by clinical data and regulatory approval as for drugs and medical devices. An example is WellDoc's BlueStar Rx mobile app, which was cleared by the FDA as a prescription-only app to support the management of type 2 diabetes. Another version without the bolus calculator, called BlueStar, was approved for direct sale 
without prescription (i.e. two versions are offered, allowing the company to offer the product through more channels). Both BlueStar and BlueStar Rx analyse diabetes data entered by the patient, comparing past data trends to form personalised guidance and creating a summary of curated data analytics to the healthcare team for clinical decision support, but the nonprescription version will not feature the insulin calculator that the full version does. In essence, digital therapeutics such as BlueStar Rx focus on delivering clinical outcomes and are regulated by the FDA. On the other hand, mobile health apps, especially those that do not provide clinical recommendations, are largely not.

Whether a mobile app has regulatory clearance/approval or not, we believe that all clinical performance claims made by 'digital health technology' should be backed by clinical evidence and real-world performance/outcomes. Real-world data and real-world evidence have been increasingly recognised by regulatory bodies, including the FDA, to enhance clinical research and support regulatory decision making for drugs, biologics and devices [51-52], and thus the same should be done for mobile apps. The FDA has published a Digital Health Innovation Action Plan that outlines a reimagined approach to foster digital health innovation while continuing to protect and promote public health [47]. This effort includes three goals:

1. Providing guidance to provide clarity on the medical software provisions of the 21st Century Cures legislation;

2. Launching an innovative pilot precertification programme (called the FDA Pre-Cert for Software) to develop a new approach to digital health technology oversight; and

3. Building expertise within the agency (including recruitment of additional dedicated and specialised staff).

In recent months, the FDA has further developed its Digital Health Software Precertification Pilot Program (Pre-Cert) with the goal of developing a more tailored pathway that enhances safety and effectiveness of such devices while supporting the innovation and availability of high-quality digital health tools. This programme will allow the FDA to first look at the company, rather than primarily at the digital health software product being submitted, in order to expedite product reviews from vetted 'excellent' companies [53]. The components of the Pre-Cert programme are:

1. Excellence appraisal and certification: Evaluating organisational excellence based on five criteria for quality and organisational excellence principles: (i) product quality, (ii) patient safety, (iii) clinical responsibility, (iv) cybersecurity responsibility, and (v) proactive culture.

2. Review determination: A risk-based framework for precertified organisations is to be established to determine the premarket review pathways for their products.
Incorporating principles from the International Medical Device Regulators Forum (IMDRF)'s Software as a Medical Device (SaMD) (discussed in the next section), the final framework for each SaMD will be based on the state of the healthcare condition addressed, the significance of the information provided to support healthcare decisions, and descriptions of the core functionality and device.

3. Streamlined review: FDA review of the information received, which is made streamlined because (1) and (2) provide information that does not need to be submitted again.

4. Real-world performance: Post-launch product-monitoring efforts on product-specific real-world performance analytics (RWPA). RWPA will consist of real-world health analytics (RWHA: human factors and usability engineering, clinical safety and health benefits), user experience analytics (UXA: user satisfaction, engagement, feedback channels and issue resolution) and product performance analytics (PPA: cybersecurity and product performance; data to be collected by the respective company) [54].

The current pilot Pre-Cert programme, which remains in a test plan phase, includes nine software companies (Apple, Fitbit, Johnson \& Johnson, Pear Therapeutics, Phosphorus, Roche, Samsung, Tidepool and Verily), seven of which have software relevant to diabetes. In 2019, the FDA will test the effectiveness of the Pre-Cert programme by reviewing a number of SaMD products under a traditional de novo pathway and, in parallel, a Pre-Cert pathway to see if the agency gets the same result [48].

\section{Guidance from other bodies}

IMDRF The IMDRF, founded in 2011, is a group of international medical device regulators whose goals are to build on the work of the Global Harmonization Task Force (GHTF) and accelerate medical device regulatory harmonisation and convergence [55]. Members include officials from the FDA, European Commission, Australian Therapeutic Goods Administration (TGA), Chinese National Medical Product Association (NMPA) and Russian Federal Service for Surveillance in Healthcare (Roszdravnadzor).

The group has released several influential documents. Among them is one released in 2014 that introduces a foundational approach, harmonised vocabulary, and general and specific considerations for manufacturers, regulators and consumers to consider in the context of SaMD [56]. In 2015, the group published another document to help manufacturers and regulators attain a common understanding and vocabulary for the application of medical device quality 
management system requirements for SaMD [57]. In 2017, the IMDRF published guidance on gathering evidence for clinically meaningful SaMDs, elaborating on valid clinical association, analytical validation and clinical validation [58]. These efforts on the global harmonisation of medical device regulatory processes, including those governing digital health technology, provide guiding principles as a template for other regulatory agencies to incorporate into their respective frameworks.

WHO In 2018, WHO published 'Classification of digital health interventions v1.0' with similar aims of providing governments, technologists, clinicians, researchers and other communities in digital health a shared and standardised language for assessing digital health interventions. The document organises digital health technologies into interventions for clients, HCPs, health system or resource managers, and data services. It also presents health system challenges and digital health interventions to address them. For example, the challenge of HCPs losing clients to follow-up can be addressed by sending alerts and reminders; this intervention is categorised under 'client communication systems' [59]. This newly available resource provides several examples of current apps and their uses and, more importantly, a solid framework to underpin future developments in digital technology.

Nationwide healthcare service At least one nationwide healthcare service now provides a digital health apps certification programme. The UK's National Health Service (NHS) describes its process, which involves app providers to show evidence that their products pass tests in outcomes, clinical safety, data protection, security, usability and accessibility, interoperability and technical stability [60]. The NHS has so far listed 13 apps that are 'safe and secure' for the management of diabetes: Changing Health, GDmHealth, Liva UK, Low Carb Program, mapmydiabetes, Mumoactive, My Diabetes My Way, My Health Fabric, my mhealth: myDiabetes, nujjer, OurPath, Oviva and Sugarmedown [61]. This is the only database dedicated solely to apps approved by a regulatory body of which we are aware.

\section{Issues faced by the diabetes community}

Although the rapid growth of digital health apps potentially brings great benefit, because this field is still in its infancy, there are also questions and challenges: for example, how physicians and other HCPs can maintain an adequate understanding of commonly used apps in order to provide guidance to people with diabetes, and how data can be kept confidential and secure.
Below we outline nine major issues that need to be addressed by regulatory authorities, policymakers, professional organisations, researchers, product manufacturers and HCPs.

Availability of evidence Although there are almost half a million mobile health apps available for download, there are far fewer RCTs, case-control studies and cohort studies that have evaluated whether app-based interventions improve health-related behaviours. One of the reasons there are so few published RCTs of digital health is that a product is never 'frozen' in time like a medication; program developers are constantly improving their apps. Even a 3-month RCT is likely to have at least a 2-year timeline from conception to publication; a long period of time in a fastdeveloping area. What should also be kept in mind is that RCTs on digital health apps, are, by nature, never blinded, so a placebo effect cannot be ruled out. Another reason for the relatively few RCTs is that the typically lower commercial value and shorter life cycles of these products does not support the high cost and time involved in conducting RCTs. As a result, developing apps to be used in medical studies may be a less attractive business model for mobile health app developers.

In 2016, Zhao and colleagues searched for peerreviewed articles in English, published from January 2010 to June 2015, on app-based health interventions targeted at adult populations. While their initial search returned over 3300 articles, the exclusion of qualitative studies and those in which mobile apps were not the primary intervention tool resulted in a final 23 articles from which primary or secondary outcomes for analysis could be extracted [62]. This small number starkly contrasts with the number of mobile health apps available for download. Of these 23 articles, there were only ten described studies relevant to diabetes management. Four of these ten provided interventions intended to improve lifestyle (e.g. physical activity, weight control and diet control), and three aimed to improve medication management. However, only two actually measured changes during a lifestyle intervention, and only one was specifically targeted at diabetes management. Several of the apps assessed in this study improved short-term adherence and enhanced intervention effectiveness, but many others yielded no effect. Zhao and others concluded that their results provided a snapshot of the current evidence of effectiveness for health-related apps, but large-sample, high-quality, adequately powered RCTs are required.

Similarly, in 2016, Drincic and others reviewed mobile medical apps that were commercially available to people with diabetes in the USA or EU. They found only 14 apps with clinical outcomes data published in peer-reviewed literature or that have been cleared by the FDA in the USA or received a 
CE mark in Europe. Drincic and others found these apps to positively affect outcomes, such as $\mathrm{HbA}_{1 \mathrm{c}}$, hypoglycaemia incidence and diabetes self-care measures, in the short term. However, more data and long-term studies are needed [31].

More recently, a 2018 comprehensive study for the US Agency for Healthcare Research and Quality found only 11 RCTs [clinical vs control] reporting health outcomes among the hundreds of commercially available apps for diabetes self-management. Of these 11 RCTs, only five were associated with clinically significant but small improvements in $\mathrm{HbA}_{1 \mathrm{c}}$. None of the studies demonstrated improvements in quality of life, blood pressure, weight or BMI. Methodological issues included limited duration (2-12 months), potential confounding by other co-interventions, and inconsistency in the reporting of randomisation, allocation, masking of outcomes assessment and method of analysis in relation to dropouts. None of the studies were considered to be high quality [63].

Thus, while the available studies of app-based interventions show promise for promoting healthy behaviour and managing complex diseases, such as diabetes, they are extremely limited in both quantity and quality. The studies previously mentioned in this section all report their respectively assessed apps as improving or showing promise in improving short-term outcomes. However, all of these studies also conclude that more rigorous, larger sample and longer-term RCTs are required to distinguish the effect of these apps from possible concomitant effects. In principle, well-designed studies with larger sample sizes and of longer duration are needed to gather and assess evidence of sustainable effectiveness over time.

Adequate information and training Beyond the field of diabetes, evidence-based apps are available as clinical decisionmaking tools for HCPs, with a scope that includes disease diagnosis, medical calculators, literature search and reference drug information [64]. With thousands of apps currently being developed and updated, issues arise. These issues include how to keep HCPs up to date with the apps most appropriate to use, how to support people with diabetes to use these digital tools, and how to ensure that using them will result in benefit rather than harm. Although it is important for HCPs to stay up to date on the digital health app landscape, we acknowledge that it is unrealistic for HCPs to meet this expectation on top of their high workload burden. As a result, other stakeholders in the diabetes community should work with and alongside HCPs in addressing this issue.

Accuracy, clinical validity and quality Because the majority of mobile health apps are not subject to regulation, data for assessment of accuracy, defined as the ability to correctly differentiate patient and healthy cases (the sum of true positive and true negative cases divided by the sum of all cases) [65], often may not be available. Patient involvement and selfmanagement are the key to diabetes care, but there is a fine line between empowerment and unregulated harm. For example, potentially questionable data and/or medical opinion from a mobile health app can place a burden on a consultation if the information provided does not align with clinical guidelines in disease management [66].

A number of studies have evaluated the accuracy of mobile medical and health apps, though there are few studies that focus on diabetes health apps. Chavez and others analysed the 89 most popular free English language diabetes apps by each app's level of engagement, functionality, aesthetics, information and number of diabetes-specific management tasks met. Using the Mobile App Rating Scale (MARS), they found that while this subset of mobile health apps ranked 'acceptable-good' in engagement, functionality and aesthetics, they ranked 'poor-acceptable' in information, app quality score and app subjective score [67]. Bierbrier and others evaluated the accuracy of 14 smartphone medical calculation apps aimed at internists, including those that calculated the severity or likelihood of liver disease or of having a pulmonary embolism. Of 1240 calculations run on these apps, $98.6 \%$ were accurate, with six of the 14 functions assessed as $100 \%$ accurate. Although errors were few overall, some were clinically significant. The authors point out that in the absence of regulation, the responsibility for any adverse consequences of using these apps falls on the individual clinician [68].

In addition, a 2018 study by Lum and others pointed out the need for quality assurance mechanisms for diabetes apps to support people with diabetes. Of the approximately 370 diabetes apps that met the researchers' criteria for blood glucose self-management (blood glucose level recording; goal setting for blood glucose levels and $\mathrm{HbA}_{1 \mathrm{c}}$; reminders, alerts, and action prompts; and patient education on hypoglycaemia and hyperglycaemia management), the majority did not provide real-time decision support or situation-specific education on blood glucose self-management. All of these apps recorded blood glucose levels. However, only about a third had goal setting and reminders to measure blood glucose and record $\mathrm{HbA}_{1 \mathrm{c}}$. Approximately a third of apps alerted users to hypoglycaemia or hyperglycaemia, and only $10 \%$ of apps educated users on blood glucose management [69].

Thus, greater scrutiny is needed to oversee the accuracy, clinical validity and quality of mobile health apps to protect patient safety. Apps that can be used by adolescents or parents for their children, as well as during pregnancy or old age, have to ensure that the advice given is suitable for the target age group. In addition, apps should clearly define the user group.

Another factor that should be considered is the standardisation of language and presentation (e.g. blood sugar, 
time in range, standard deviation, BMI). Setting standards for how information is presented would lead to fewer errors in translation and interpretation from app to HCP to patient.

Technological issues Technological issues apply to diabetes digital health apps. These include the maintenance of mobile apps so that they are up to date with the latest technological platforms and operating systems and free of bugs that degrade app performance. App developers need to carefully consider battery usage, input/output ports (e.g. USB port, headphone jack, lightning port), and the impact of inconsistent illumination, mobile device cases, and inconsistent resolution with smartphone cameras [19]. In addition, the speed at which mobile app versions are released or new features are rolled out, as well as the tolerance level of acceptable error within a release, is far greater than those of medical technologies. From the user perspective, this provides greater medical choice in medical apps, but makes it more challenging to find and ensure acceptable performance among many apps of varying quality.

Interoperability and standardisation Consumers use a variety of mobile technological platforms, including Android and Apple iOS. Android and Apple iOS are the dominant platforms in the US market, with a keyword search for 'diabetes' performed in the Apple and Google Play stores in 2017 identifying 246 available apps for Android and 100 for Apple iOS [70]. As of 2012, more than 75\% of physicians in the USA use Apple iOS devices [71]. However, where these apps are available for less popular platforms, app developers should ensure they operate consistently to the same standard.

It is also important that data recorded in health apps be easily transmitted from smartphones to other platforms, such as electronic health records for sharing with HCPs. An example is Apple Health, which is a health informatics mobile app that functions as a central repository for health information. Apple Health can be integrated into multiple mobile health and fitness apps on Apple products, and record and share health data [72]. An example of an app integrated with Apple Health is Tidepool Mobile, which can connect to Apple Health and show data from users' insulin pumps, CGM systems and sources outside of Dexcom devices [73]. Google Fit is an approximate equivalent to Apple Health for the Android platform [74]. Advances in integration and automation of data collection have come far, and we anticipate these advances to continue.

Differences among populations In 2017, an estimated 12 million people $\geq 65$ years of age and 193,000 people $<20$ years of age had diabetes in the USA [75]. The differences in these two populations are important because younger populations (usually with type 1 diabetes) are typically more proficient at using smartphones than older populations. Consequently, apps targeted for older people with diabetes must be designed with their expected level of technology proficiency kept in mind. In addition, currently available diabetes management apps may not be available in languages other than English or accessible to people with certain physical or mental disabilities (e.g., colour blindness, blindness, hearing impairment). Furthermore, those from remote regions and areas of extreme socioeconomic deprivation may not have access to smartphone technology. The cost of obtaining and activating a smartphone, not to mention the cost of apps that are not free of charge to download, may be a significant barrier on top of the premium prices paid for most branded diabetes drugs [71].

To date, app developers have made strides in increasing the durability of benefits by utilising 'gamification' to encourage long-term behaviour changes and adherence to diabetes management principles. An example is a patientengagement programme, in which 'points' can be earned for time spent in range for blood glucose measurements and redeemed for pharmacy rebates, HCP visits or other benefits. This approach can also be used to encourage health outcomes [76]; examples exist within mySugr and Medtronic Inner Circle [77-78]. In addition, United Healthcare launched its Motion program, offering up to $\$ 4$ per day for beneficiaries who meet activity goals [79]. While gamification can certainly incentivise consumers to better monitor their health, it is not a 'one-size-fits-all' solution. Such programs may have the drawback of leaving behind those who are in the most need of help, such as those experiencing socioeconomic deprivation. Furthermore, there is no clear proof that gamification improves outcomes and results in long-term changes in health [80].

Another potential way to engage consumers, particularly those of older populations, is to involve Centers for Medicare \& Medicaid Services (CMS) reimbursement. Reimbursement policies in the USA could include, for example, sharing of health data in place of an office visit or sharing of CGM data.

Appropriate role of HCPs HCPs play an important role in advancing the use of diabetes mobile health apps. While a mobile health app cannot (and should not) replace an HCP, mobile health apps can certainly supplement and bolster medical practice.

As previously discussed, HCPs need to be supported to stay up to date on the diabetes digital app landscape. The ability to communicate regularly with people with diabetes and monitor their glycaemic data gives HCPs an unprecedented opportunity to monitor and improve quality of care and health outcomes (see text box: Consensus report recommendations).

Role of professional organisations Professional organisations, such as the ADA and EASD, play an important role in shaping 
the future of healthcare. In addition to the above-mentioned efforts of WHO and IMDRF to classify digital health technology, issues remain that professional organisations need to address. We believe that the American Medical Association (AMA), the International Diabetes Federation (IDF), and many others can make a greater positive impact on patient populations worldwide in collaboration with WHO and IMDRF (see text box: Consensus report recommendations).

Data security and privacy Data security is a key aspect in a digital world, especially for medical data. Although diabetes apps primarily permit people with diabetes to monitor their own data and discuss their data with health professionals, safety regarding data security and privacy remains a risk and cybersecurity has to be ensured.

Users may believe that their health data stored in apps are private, but that is often not the case. A 2014 study of diabetes apps for Android smartphones demonstrated that diabetes apps routinely shared information with third parties [81]. Because of the potential adverse impact of sharing sensitive health data, app developers should implement and fully disclose their privacy policies to users. App developers should also allow users to have full control over what data they are willing to share with third parties. Such cybersecurity measures must be implemented to protect privacy and enhance data security so that people with diabetes have adequate privacy protection and are not judged or discriminated against based on their blood glucose levels, adherence to their care, or simply their diabetes diagnosis itself.

People with diabetes have a high need for secure information when viewing their glucose levels and insulin doses on wireless diabetes devices, such as blood glucose monitors, continuous glucose monitors, and insulin pumps. Medical devices are prone to security-breaching attacks; for example, incidents have been reported in which data from insulin pumps were accessed remotely and their function controlled without the knowledge of the user. Although there have been no publicly reported incidents of users being harmed from hacking attacks, such situations have the potential to be lifethreatening [82]. Data stored in health data apps should be sufficiently encrypted to prevent serious and malicious attacks.

An example that the cybersecurity regulation of diabetes mobile health apps could follow is the guidance by the Diabetes Technology Society on the 'Standard for Wireless Diabetes Device Security (DTSec)'. DTSec establishes a high level of assurance that electronic products deliver the security protections claimed by their developers and required by their users. A DTSec-certified product must pass evaluation by a DTSec-approved laboratory and the DTSec Working Group (DWG) before it can be listed under a publicly disclosed DTSec evaluated products list [83].

\section{Conclusions and outlook}

Digital health technology, especially digital health apps, for people with or at risk for diabetes has developed at a rapid pace and become an increasingly common aspect of diabetes care and self-management in certain populations. However, several barriers remain that prevent digital health technology from reaching its full potential to improve diabetes therapies and the lives of people affected by diabetes.

Insufficient evidence (at least from a conventional way of looking at evidence) of clinical validity, effectiveness, accuracy and safety are some of the largest issues that limit the effectiveness of diabetes digital health technology. Furthermore, poor usability resulting from technological issues, interoperability issues and differences among populations is another barrier. This web of interconnected issues cannot be solved by one party alone; rather, commitment from regulators, industry, clinical experts, and funding and patient organisations is needed for the necessary clinical evidence to be gathered.

We outline a list of recommendations for regulatory agencies, manufacturing companies, international and national professional societies, funding bodies, researchers, HCPs and people with diabetes to take into careful consideration. These can be categorised into the following themes:

- more systematic and structured guidelines for digital health app development and assessment (1a-c; 3d, e)

- improved consistency and accessibility of safety reports and app documentation (2a, b, d)

- greater investment in gathering of clinical data to provide evidence on digital health interventions $(4 a, b ; 5 a, b)$

- increased accessibility for all consumer populations to use diabetes mobile apps confidentially and securely $(2 \mathrm{c}, \mathrm{g}$; 3c)

- increased communication and cooperation across stakeholder groups (1d-g; 2e, f; 3a, b, f; 6a-c; 7a-c).

Today's world of products and services, including digital health apps, is moving towards a market of integration. Apps are converging towards a data-capturing and autoanalysed future with algorithm-based recommendations for users affecting their behaviour and decisions. We envision an ongoing role of the EASD, ADA and other professional medical associations in supporting and expanding the field of diabetes digital health technology in the march to integration and continued automation. We call upon regulatory agencies and manufacturing companies to work urgently and collaboratively with health professionals, researchers and people with diabetes to create an environment in which diabetes can be managed safely and effectively, bringing benefits to all stakeholders and the entire diabetes community. 


\section{Consensus report recommendations}

\section{Regulatory agencies should:}

a. establish and update standards to be met by digital health technology developers at premarketing and postmarketing stages, such as elements of clinically validated information (not necessarily from RCTs), service systems to support users, effectiveness variables to enhance outcomes, and functions to transmit data to other devices, while also supporting market innovation

b. provide a regulatory paradigm, such as that outlined by IMDRF, which is tailored specifically to software, taking the short product life cycle and rapid turnover of updates into account

c. provide guidance for obtaining and promoting evidence of safety, effectiveness and other performance measures

d. find ways to evaluate the security, accuracy and reliability of digital health apps (e.g. by recognising and following the DTSec model), including supporting companies (often small) to generate real-world data when they have a product that has achieved a certain standard

e. provide, publicise, and maintain a single publicly accessible international database of available digital health apps and their utility/quality, including harmonising the parameters that would measure utility/quality and how these parameters would be assessed [84]

f. publish an annual summary of regulatory activities

g. work to harmonise their activities

\section{Manufacturing companies should:}

a. comply with regulations, industry standards and best practices established for digital health app development and marketing, such as providing a regularly updated flow chart that describes the decision-making process for releasing app updates; a broader plan for software maintenance and testing; and plans for obsolescence for when a specific mobile device model or operating system for which the app has been validated is discontinued [16]

b. include sufficient documentation, training modules and help-desk resources to ensure optimal use

c. provide interfaces that are user-friendly across all demographic groups and can be personalised with real-time insights and suggestions for individual users (taking their socioeconomic status into account, especially around health literacy)

d. report all safety-related data promptly and transparently to the regulatory authorities

e. cooperate with academics and HCPs to provide balanced and adequate information for people with diabetes and package the output data in standardised formats for ease of access in electronic health records

f. enable users to opt to submit their data anonymously to track outcomes and demographics following a crowd-sourcing model

g. incorporate high degrees of data security and patient confidentiality (e.g. by adhering to the DTSec model)

\section{International and national professional societies should:}

a. bring people with diabetes, HCPs, manufacturing companies and regulatory authorities together to facilitate digital health technology interventions

b. encourage academia and medical associations to advance research in digital health app effectiveness, safety and outcomes

c. help set expectations for HCPs and consumers regarding the strengths and limitations of digital technology

d. provide evidence-based guidelines on the effectiveness of digital health interventions

e. recommend appropriate forms of structured education required for HCPs to support people with diabetes to benefit from the best digital health (HCPs cannot be trained in the use of each app; however, they can be supported in maintaining a basic understanding of what apps can do and how they are used)

f. maintain a list of endorsed apps that have passed a threshold of accuracy, dependability and ease of use for both people with diabetes and HCPs

4. International and national research funding bodies should:

a. provide or facilitate funding for well-designed independent clinical studies that measure safety, effectiveness, outcomes and use in real-world settings

b. provide or facilitate significant financial support for long-term data collection

\section{Researchers/academics should:}

a. openly report and share the patient-level results of all clinical evidence

b. develop and validate specific and appropriate patient-related outcome measures

\section{HCPs should:}

a. be knowledgeable of digital health apps and their strengths and weaknesses

b. support and inform people with diabetes on the use of digital health apps to augment diabetes management and lifestyle modification

c. use health data to improve quality of care and health outcomes

\section{Consumers of digital health apps (people with diabetes, family members, caregivers) should:}

a. consider digital health apps as a valuable addition or supplement to disease management or prevention

b. discuss with their HCPs available and appropriate digital health app options, as well as advice or counselling received from the app that affects behaviour or care decisions

c. submit app reviews, which would include information on digital health app efficacy, success, errors and malfunctions, as well as report apps that appear to be unsafe or illegally marketed, to the manufacturers and appropriate regulatory agencies and care organisations (e.g. ADA) 
Acknowledgements We would like to thank the following for their helpful comments: Adam Brown (Close Concerns), John Buse (University of North Carolina School of Medicine), Danelle Miller (Roche Diagnostics), Laleh Mohajerani (Novo Nordisk), Nick Riggall (LB Talent), Mindy Saraco (American Diabetes Association), David Strasberg (Lee Strasberg Institute) and Craig Stubing (Beta Cell podcast). We would very much like to thank Jennifer Zhao (Kinexum) for her excellent editorial help.

Funding No funding was received.

Duality of interest No honoraria were received by members of the ADAEASD Diabetes Technology Committee (AEDTC) for writing this manuscript or associated meetings. Most of the members of the AEDTC work with industry as listed below; however, the industry had no impact on the manuscript and its content.

GAF is Executive Chairman of Kinexum, which advises multiple healthproduct companies in the fields of metabolism, cardiovascular, oncology and dermatology. He was formerly the Group Leader of the Division of Metabolism and Endocrine Drug Products at the US Food and Drug Administration (FDA). JRP has served on advisory boards for companies manufacturing pharmaceuticals used in the treatment of diabetes but no digital health companies. RMB has conducted clinical trials, consulted or served on the scientific advisory boards for Abbott Diabetes Care, Becton Dickinson, Bayer, Dexcom, Eli Lilly, Johnson \& Johnson, Medtronic, Novo Nordisk, Roche Diabetes Care and Sanofi. He receives no personal income from these activities, for all payment goes to the non-profit Park Nicollet Institute. RWH coordinates the German/Austrian DPV initiative, which has been supported by Novo Nordisk, Medtronic, Roche, Boehringer-Ingelheim and Eli Lilly through institutional research grants. RWH did not receive any personal honoraria. ALP has served on advisory boards for Abbott Diabetes Care, Eli Lilly, Merck, Novo Nordisk, MannKind, Lexicon, Sanofi and Zafgen. She has received grant funding from AstraZeneca, MannKind and Dexcom. She has been on the Speaker's Bureau for Novo Nordisk. LH is partner of Profil Institut für Stoffwechselforschung in Neuss, Germany, and of ProSciento in San Diego, CA, USA. He is a member of advisory boards for Roche Diagnostics, Abbott, Zense and Medtronic.

Contribution statement All authors were responsible for drafting the article and revising it critically for important intellectual content. All authors approved the version to be published.

\section{References}

1. NCD Risk Factor Collaboration (NCD-RisC) (2016) Worldwide trends in diabetes since 1980: a pooled analysis of 751 population-based studies with 4.4 million participants. Lancet 387(10027):1513-1530

2. Gillies CL, Abrams KR, Lambert PC et al (2007) Pharmacological and lifestyle interventions to prevent or delay type 2 diabetes in people with impaired glucose tolerance: systematic review and meta-analysis. Br Med J 334(7588):299. https://doi.org/10.1136/ bmj.39063.689375.55

3. Knowler WC, Barrett-Connor E, Fowler SE et al (2002) Reduction in the incidence of type 2 diabetes with lifestyle intervention or metformin. N Engl J Med 346(6):393-403. https://doi.org/10. 1056/NEJMoa012512

4. Tuomilehto J, Lindstrom J, Eriksson JG et al (2001) Prevention of type 2 diabetes mellitus by changes in lifestyle among subjects with impaired glucose tolerance. N Engl J Med 344(18):1343-1350. https://doi.org/10.1056/NEJM200105033441801
5. Ramchandani N, Heptulla RA (2012) New technologies for diabetes: a review of the present and the future. Int $\mathrm{J}$ Pediatr Endocrinol 2012(1):28

6. Kaufman N, Khurana I (2016) Using digital health technology to prevent and treat diabetes. Diabetes Technol Ther 18(Suppl 1):S56 S68

7. WHO (2011) mHealth: new horizons for health through mobile technologies. Based on the findings of the second global survey on eHealth. Global Observatory for eHealth series - Volume 3. WHO, Geneva. Available from www.who.int/goe/publications/ goe mhealth web.pdf. Accessed 17 Sep 2018

8. Elenko E, Speier A, Zohar D (2015) A regulatory framework emerges for digital medicine. Nat Biotechnol 33(7):697-702. https://doi.org/10.1038/nbt.3284

9. Statista. Number of mHealth apps available in the Apple App Store from 2nd quarter 2015 to 3rd quarter 2018. Available from www. statista.com/statistics/779910/health-apps-available-iosworldwide/. Accessed 3 Dec 2018

10. Pohl M (2017) 325,000 mobile health apps available in 2017 Android now the leading mHealth platform. Available from https://research2guidance.com/325000-mobile-health-appsavailable-in-2017/. Accessed 3 Dec 2018

11. Martínez-Pérez B, de la Torre-Díez I, López-Coronado M (2013) Mobile health applications for the most prevalent conditions by the World Health Organization: review and analysis. J Med Internet Res 15(6):e120. https://doi.org/10.2196/jmir.2600

12. American Association of Diabetes Educators (2008) AADE7 selfcare behaviours. Diabetes Educ 34:445-449

13. Kebede MM, Pischke CR (2019) Popular diabetes apps and the impact of diabetes app use on self-care behaviour: a survey among the digital community of persons with diabetes on social media. Front Endocrinol (Lausanne) 10:135. https://doi.org/10.3389/ fendo.2019.00135

14. Bergenstal RM, Johnson M, Powers MA et al (2008) Adjust to target in type 2 diabetes. Diabetes Care 31(7):1305-1310. https:// doi.org/10.2337/dc07-2137

15. Conroy DE, Yang CH, Maher JP (2014) Behavior change techniques in top-ranked mobile apps for physical activity. Am J Prev Med 46(6):649-652. https://doi.org/10.1016/j.amepre.2014.01.010

16. WellDoc (2017) WellDoc Receives FDA 510(k) Clearance to Offer a Non-Prescription Version of BlueStar ${ }^{\circledR}$ Digital Therapeutic for Type 2 Diabetes. Available from www.welldoc.com/news/ welldoc-receives-fda-510k-clearance-to-offer-a-non-prescriptionversion-of-bluestar-digital-therapeutic-for-type-2-diabetes/. Accessed 6 Sep 2019

17. Brown A. Can diabetes apps make our lives easier? Lecture presented at: ADA Scientific Sessions; 23 June 2018; Orlando, FL. Available from https://diatribe.org/Apps2018. Accessed 3 Oct 2019

18. Cision PR Newswire (2019) Hygieia receives 510(k) clearance from FDA for phone app that enhances company's d-Nav insulin guidance service. Available from www.prnewswire.com/newsreleases/hygieia-receives-510k-clearance-from-fda-for-phone-appthat-enhances-companys-d-nav-insulin-guidance-service300797091.html. Accessed 26 February 2019

19. Schwartz N. FDA perspective: mobile medical apps in diabetes management. Lecture presented at: Diabetes Technology Meeting; 9 Nov 2018; Bethesda, MD

20. Companion Medical. InPen. Available from www. companionmedical.com/InPen/. Accessed 7 Dec 2018

21. Dexcom. Dexcom Clarity. Available from https://clarity.dexcom. com. Accessed 13 Feb 2019

22. Medtronic. Sugar.IQ ${ }^{\mathrm{TM}}$ Diabetes Assistant $\mid$ Exclusive to Guardian ${ }^{\mathrm{TM}}$ Connect CGM. Available from www.medtronicdiabetes.com/ products/sugar.iq-diabetes-assistant. Accessed 13 Feb 2019

23. US Food \& Drug Administration. What is the pancreas? What is an artificial pancreas device system? Available from www.fda.gov/ 
medical-devices/artificial-pancreas-device-system/what-pancreaswhat-artificial-pancreas-device-system. Accessed 12 Jun 2019

24. diaTribe. The FDA Approves Medtronic's MiniMed 670G Hybrid Closed Loop System. Available from https://diatribe.org/fdaapproval-medtronic-minimed-670g-hybrid-closed-loop-system. Accessed 3 Oct 2019

25. Bankmycell.com. How many phones are there in the world? 1 billion more mobile connections than people worldwide. Available from www.bankmycell.com/blog/how-many-phonesare-in-the-world\#targetText=Accordingto Statista, thereis, populationhaveasmartphonetoday. Accessed 6 Sep 2019

26. Hood M, Wilson R, Corsica J, Bradley L, Chirinos D, Vivo A (2016) What do we know about mobile applications for diabetes self-management? A review of reviews. J Behav Med 39(6):981994. https://doi.org/10.1007/s10865-016-9765-3

27. Frøisland DH, Årsand E (2015) Integrating visual dietary documentation in mobile-phone-based self-management application for adolescents with type 1 diabetes. J Diabetes Sci Technol 9(3): 541-548. https://doi.org/10.1177/1932296815576956

28. Turner-McGrievy GM, Beets MW, Moore JB, Kaczynski AT, BarrAnderson DJ, Tate DF (2013) Comparison of traditional versus mobile app self-monitoring of physical activity and dietary intake among overweight adults participating in an mHealth weight loss program. J Am Med Inform Assoc 20(3):513-518. https://doi.org/ 10.1136/amiajnl-2012-001510

29. Huang Z, Tao H, Meng Q, Jing L (2015) Management of endocrine disease. Effects of telecare intervention on glycemic control in type 2 diabetes: a systematic review and meta-analysis of randomized controlled trials. Eur J Endocrinol 172(3):R93-R101. https://doi. org/10.1530/EJE-14-0441

30. Arnhold M, Quade M, Kirch W (2014) Mobile applications for diabetics: a systematic review and expert-based usability evaluation considering the special requirements of diabetes patients age 50 years or older. J Med Internet Res 16(4):e104

31. Drincic A, Prahalad P, Greenwood D, Klonoff DC (2016) Evidence-based mobile medical applications in diabetes. Endocrinol Metab Clin N Am 45(4):943-965. https://doi.org/10. 1016/j.ecl.2016.06.001

32. US Food \& Drug Administration (2019) Policy for device software fuctions and mobile medical applications. Available from www.fda. gov/media/80958/download. Accessed 8 Nov 2019

33. Kerr D, Gabbay RA, Klonoff DC (2018) Finding real value from digital diabetes health: is digital health dead or in need of resuscitation? J Diabetes Sci Technol 12(5):911-913. https://doi.org/10. 1177/1932296818771200

34. Schuren J, Califf RM (2016) Need for a national evaluation system for health technology. JAMA 316(11):1153-1154

35. Sherr JL, Tauschmann M, Battelino T et al (2018) ISPAD clinical practice consensus guidelines 2018: diabetes technologies. Pediatr Diabetes 19(Suppl 27):302-325

36. European Commission. Guidance document medical devices scope, field of application, definition - qualification and classification of stand alone software - MEDDEV 2.1/6. Available from http://ec.europa.eu/DocsRoom/documents/17921/attachments/1/ translations. Updated July 2016. Accessed 19 Sep 2018

37. European Commission. Transformation of health and care in the digital single market. Available from https://ec.europa.eu/digitalsingle-market/en/european-policy-ehealth. Accessed 19 Sep 2018

38. European Commission. EU health policy. Available from https://ec. europa.eu/health/policies/overview_en. Accessed 19 Sep 2018

39. Kramer DB, Xu S, Kesselheim AS (2012) Regulation of medical devices in the United States and European Union. N Engl J Med 36(9):848-855

40. European Commission (2016) The "Blue Guide" on the implementation of EU products rules 2016. Available from https://eur-lex.
europa.eu/legal-content/EN/TXT/PDF/?uri=CELEX: 52016XC0726(02)\&rid=7. Accessed 22 Sep 2018

41. Van Norman GA (2016) Drugs and devices: comparison of European and U.S. approval processes. JACC Basic Transl Sci 1(5):399-412. https://doi.org/10.1016/j.jacbts.2016.06.003

42. European Commission. Medical Devices Regulatory Framework. Available from https://ec.europa.eu/growth/sectors/medicaldevices/regulatory-framework_en. Accessed 13 Feb 2019

43. Läkemedelsverket Medical Products Agency. Medical information systems - guidance for qualification and classification of standalone software with a medical purpose. Läkemedelsverket Medical Products Agency, Uppsala. Available from https:// lakemedelsverket.se/upload/eng-mpa-se/vagledningar_eng/ medical-information-system-guideline.pdf. Accessed 20 Sep 2018

44. European Network of Research Ethics Committee. The Act on Medical Devices (Medical Devices Act) (Medizinproduktegesetz - MPG) (especially $\S \S 19-24)$. Available from www.eurecnet.org/ legislation/germany.html. Accessed 20 Sep 2018

45. US Food \& Drug Administration. Mobile medical applications. Available from www.fda.gov/medical-devices/digital-health/ mobile-medical-applications. Accessed 6 Sep 2019

46. US Food \& Drug Administration. 21st Century Cures Act. Available from www.fda.gov/regulatoryinformation/ lawsenforcedbyfda/significantamendmentstothefdcact/ 21stcenturycuresact/default.htm. Accessed 19 Sep 2018

47. US Food \& Drug Administration. Digital health. Available from www.fda.gov/MedicalDevices/DigitalHealth/default.htm. Accessed 19 Sep 2018

48. US Food \& Drug Administration. National evaluation system for health technology (NEST). Available from www.fda.gov/aboutfda/ centersoffices/officeofmedicalproductsandtobacco/cdrh/ cdrhreports/ucm301912.htm. Accessed 13 Feb 2019

49. Shuren J (2018) Changing times at the FDA. Lecture presented at: Diabetes Technology Meeting, 9 Nov 2018, Bethesda, MD

50. Kvedar JC, Fogel AL, Elenko E, Zohar D (2016) Digital medicine's march on chronic disease. Nat Biotechnol 34(3):239-246

51. US Food \& Drug Administration (2019) Submitting documents using real-world data and real-world evidence to FDA for drugs and biologics: guidance for industry. Available from www.fda. gov/media/124795/download. Accessed 13 Jun 2019

52. US Food \& Drug Administration. Use of real-world evidence to support regulatory decision-making for medical devices: guidance for industry and food and drug administration staff. Available from www.fda.gov/media/99447/download. Published 31 Aug 2017. Accessed Jun 13, 2019

53. Brown A, Carracher A, Kurian M, et al. JPM 2019: January 7-10; San Francisco, CA; Day \#2 Highlights - Draft. Available from www.closeconcerns.com/knowledgebase $/ \mathrm{r} / 5 \mathrm{a} 490 \mathrm{a} 4 \mathrm{~b}$. Updated 8 Jan 2019. Accessed 23 Jan 2019

54. US Food and Drug Administration. Digital health software precertification (Pre-Cert) program. Available from www.fda.gov/ MedicalDevices/DigitalHealth/DigitalHealthPreCertProgram/ default.htm. Accessed 13 Jun 2019

55. International medical device regulators forum. Available from www.imdrf.org. Accessed 20 Sept 2018

56. IMDRF SaMD Working Group (2014) Final document: "Software as a Medical Device": possible framework for risk categorization and corresponding considerations. Available from www.imdrf.org/ docs/imdrf/final/technical/imdrf-tech-140918-samd-frameworkrisk-categorization-141013.pdf. Accessed 13 Feb 2019

57. IMDRF SaMD Working Group (2015) Proposed document: software as a medical device (SaMD): application of quality management system. Available from www.imdrf.org/docs/imdrf/final/ technical/imdrf-tech-151002-samd-qms.pdf. Accessed 3 Oct 2019

58. International Medical Device Regulators Forum (2017) Software as a medical device (SAMD): clinical evaluation. Guidance for 
Industry and Food and Drug Administration Staff. Available from https://www.fda.gov/media/100714/download. Accessed 3 Oct 2019

59. World Health Organization (2018) Classification of digital health interventions v1.0: A shared language to describe the uses of digital technology for health. Available from https://apps.who.int/iris/ bitstream/handle/10665/260480/WHO-RHR-18.06-eng.pdf; jsessionid=CE0199154B170C471D487632C549A142? sequence $=$ 1. Accessed 20 Sep 2018

60. National Health Service. "How we access apps". Available from https://apps.beta.nhs.uk/how-we-assess-apps/. Accessed 24 Jan 2019

61. National Health Service. Diabetes-related apps. Available from https://apps.beta.nhs.uk/category/diabetes/. Accessed 24 Jan 2019

62. Zhao J, Freeman B, Li M (2016) Can mobile phone apps influence people's health behavior change? An evidence review. J Med Internet Res 18(11):e287. https://doi.org/10.2196/jmir.5692

63. Veazie S, Winchell K, Gilbert J, et al (2018) Mobile applications for self-management of diabetes. Technical Brief No. 31. (Prepared by the Scientific Resource Center under Contract Nos. 290- 20120004-C and 290-2017-00003-C.) AHRQ Publication No. 18EHC010-EF. Agency for Healthcare Research and Quality, Rockville

64. Ventola CL (2014) Mobile devices and apps for health care professionals: uses and benefits. P T 39(5):356-364

65. Baratloo A, Hosseini M, Negida A, el Ashal G (2015) Part 1: simple definition and calculation of accuracy, sensitivity and specificity. Emerg (Tehran) 3(2):48-49

66. Hogan NM, Kerin MJ (2012) Smart phone apps: smart patients, steer clear. Patient Educ Couns 89(2):360-361. https://doi.org/10. 1016/j.pec.2012.07.016

67. Chavez S, Fedele D, Guo Y, Bernier A, Smith M, Warnick J, Modave F (2017) Mobile apps for the management of diabetes. Diabetes Care 40(10):e145-e146. https://doi.org/10.2337/dc170853

68. Bierbrier R, Lo V, Wu RC (2014) Evaluation of the accuracy of smartphone medical calculation apps. J Med Internet Res 16(2): e32. https://doi.org/10.2196/jmir.3062

69. Lum E, Jimenez G, Huang Z et al (2019) Decision support and alerts of apps for self-management of blood glucose for type 2 diabetes. JAMA 321(15):1530-1532. https://doi.org/10.1001/ jama.2019.1644

70. Izahar S, Lean QY, Hameed MA et al (2017) Content analysis of mobile health applications on diabetes mellitus. Front Endocrinol (Lausanne) 8:-318

71. Ristau RA, Yang J, White JR (2013) Evaluation and evolution of diabetes mobile applications: key factors for health care professionals seeking to guide patients. Diabetes Spectrum 26(4):211215
72. Apple. Health. Available from www.apple.com/ios/health/. Accessed 3 Oct 2019

73. Tidepool. Tidepool Mobile. Available from https://tidepool.org/ users\#mobile. Accessed 13 Feb 2019

74. Google. Google Fit. Available from www.google.com/fit/. Accessed 13 Feb 2019

75. Department of Health and Human Services, Centers for Disease Control and Prevention (2017) National Diabetes Statistics Report, 2017: estimates of diabetes and its burden in the United States. CDC, Atlanta

76. Ahn D. Advances in BGM logbooks and apps. Lecture presented at: Diabetes Technology Meeting, 8 Nov 2018, Bethesda, MD

77. Rose KJ, König M, Wiesbauer F. Evaluating success for behavorial change in diabetes via mHealth and gamification: mySugr's keys to retention and patient engagement. Poster presented at: Advanced Technologies and Treatments for Diabetes; 27 Feb - 3 Mar, 2013; Paris, France. Available from https://assets.mysugr.com/website/ mysugr.com-wordpress/uploads/2017/03/attd-2013-poster.pdf. Accessed 3 Oct 2019

78. Medtronic (2018) Medtronic launches patient engagement program that leverages gamification to help motivate and engage patients living with diabetes. Available from http://newsroom.medtronic. com/phoenix.zhtml?c=251324\&p=irol-news Article \&ID= 2363121. Accessed 24 Jan 2018

79. Levine B, Brown A, and Close K (2018) Close Concerns. UnitedHealthcare adds Apple Watch to Motion physical activity incentives program; people with diabetes $40 \%$ more likely to enroll in Motion. Available from www.closeconcerns.com/ knowledgebase/r/96d89360. Accessed 24 Jan 2019

80. Sardi L, Idri A, Fernandez-Aleman JL (2017) A systematic review of gamification in e-Health. J Biomed Inform 71:3148

81. Blenner SR, Köllmer M, Rouse AJ, Daneshvar N, Williams C, Andrews LB (2016) Privacy policies of android diabetes apps and sharing of health information. JAMA 315(10):1051-1052. https:// doi.org/10.1001/jama.2015.19426

82. Klonoff DC (2015) Cybersecurity for connected diabetes devices. J Diabetes Sci Technol 9(5):1143-1147. https://doi.org/10.1177/ 1932296815583334

83. Diabetes Technology Society (2017) Standard for wireless diabetes device security (DTSec). Available from www.diabetestechnology. org/dtsec/DTSecStandard.pdf. Accessed 16 Nov 2018

84. Wyatt JC (2018) How can clinicians, specialty societies and others evaluate and improve the quality of apps for patient use? BMC Med $16: 225$

Publisher's note Springer Nature remains neutral with regard to jurisdictional claims in published maps and institutional affiliations. 